\title{
Correct Diagnosis of Vascular Encasement and Longitudinal Extension of Hilar Cholangiocarcinoma by Four-Channel Multidetector-Row Computed Tomography
}

\author{
Tadayuki OKumoto, ${ }^{1}$ Akihiro Sato, ${ }^{2}$ Takayuki Yamada, ${ }^{1}$ Kei Takase, ${ }^{1}$ \\ Toshio Matsuhashi, ${ }^{1}$ Masashi Tsuda, ${ }^{1}$ Kazumasa SeiJI, ${ }^{1}$ Tadashi Ishibashi, ${ }^{3}$ \\ Shuichi Higano, ${ }^{1}{ }^{\text {Yu Katayose, }}{ }^{4}$ Michiaki Unno ${ }^{4}$ and Shoki Takahashi ${ }^{1}$ \\ ${ }^{1}$ Department of Diagnostic Radiology, Tohoku University Graduate School of Medicine, Sendai, Japan \\ ${ }^{2}$ Department of Radiology, Sendai Medical Center, Sendai, Japan \\ ${ }^{3}$ Course of Health Sciences, Tohoku University Graduate School of Medicine, Sendai, Japan \\ ${ }^{4}$ Department of Gastroenterological Surgery, Tohoku University Graduate School of Medicine, Sendai, Japan
}

\begin{abstract}
Accurate diagnosis of local invasion of hilar cholangiocarcinomas is challenging due to their small size and the anatomic complexity of the hepatic hilar region. On the other hand, the correct diagnosis of local invasion is essential for assuring the possibility of curative surgery. The purpose of this study was to evaluate the feasibility of four-channel multidetector-row computed tomography (MDCT) in the assessment of vascular and bile duct involvement, by which we could obtain useful information for the surgical management of hilar cholangiocarcinoma. The subjects were 18 patients for whom the extent of tumor invasion was surgically and pathologically confirmed. All patients underwent preoperative multiphasic CT scanning by MDCT. Arterial and portal dominant phases were acquired using a detector configuration of $1.25 \mathrm{~mm} \times 4 \mathrm{~mm}$, and both axial and multiplanar reconstructed images were interpreted. Longitudinal extension was evaluated up to second-order branches. Vascular invasion is considered to be the degree of tumor contiguity to the hepatic arteries and portal vein and was graded by CT. The longitudinal extension was correctly diagnosed in 14 patients $(77.8 \%)$. Hepatic artery invasion was correctly diagnosed in 17 patients with sensitivity of $100 \%$ and specificity of $90 \%$, respectively. Portal vein invasion was correctly diagnosed in 47 of 51 branches with sensitivity and specificity of $92.3 \%$ and $90.2 \%$, respectively. Multiplanar reconstructed images contributed to the correct diagnosis for both vascular encasement and longitudinal tumor extension. In conclusion, MDCT is useful in preoperative evaluation of hilar cholangiocarcinoma, especially when combined with multiplanar reconstructed images. - Hilar cholangiocarcinoma; preoperative evaluation; diagnostic radiology; multidetector-row computed tomography; multiplanar reconstruction.
\end{abstract}

Tohoku J. Exp. Med., 2009, 217 (1), 1-8. 두 2009 Tohoku University Medical Press

Hilar cholangiocarcinoma represents two-thirds of cholangiocarcinomas (Nakeeb et al. 1996). It has long been considered as an incurable disease because negative surgical margins are often difficult to achieve due to local invasion and the anatomical complexity of the hepatic hilum. However, surgical management of this disease has improved over the past two decades, owing to advances in surgical techniques, perioperative management, and diagnostic imaging (Saldinger et al. 2000; Figueras et al. 2000; Hemming et al. 2005).

Since the likelihood of long-term survival is highly associated with a margin-negative resection, preoperative imaging of local tumor extension is essential for surgical planning. Of the local factors associated with hilar cholangiocarcinoma, vascular encasement and longitudinal exten- sion along the bile duct are regarded as the two most important factors in determining resectability.

Although there have been several studies on the preoperative assessment of resectability of hilar cholangiocarcinoma by multidetector-row computed tomography (MDCT) (Lee et al. 2006; Chen et al. 2006), these studies used relatively thick section images $(2.5 \mathrm{~mm})$ with no multiplanar reformation or overlap reconstruction for evaluating the biliary tree.

The purpose of this study was therefore to evaluate the diagnostic accuracy of four-channel MDCT using thin sections $(1.25 \mathrm{~mm})$ with multiplanar reformation in the preoperative assessment of vascular encasement and longitudinal extension of the cholangiocarcinoma.

Received June 9, 2008; revision accepted for publication November 25, 2008

Correspondence: Tadayuki Okumoto, MD, Department of Diagnostic Radiology, Tohoku University Graduate School of Medicine, 1-1

Seiryo-machi, Aoba-ku, Sendai 980-8574, Japan.

e-mail: okumoto@rad.med.tohoku.ac.jp 


\section{Materials And Methods}

Study Population

During January 2000-December 2002, 38 consecutive patients were admitted to our institute for the treatment of hilar cholangiocarcinoma. Of these, 20 patients were not operated on, including 13 with apparent local invasion (e.g., main portal vein invasion), 5 with distant metastases, and 2 with insufficient liver function. The 18 patients who underwent surgical resection comprised 11 men and 7 women with a mean age of 65.1 years (range: $56-80$ years).

All 18 patients had obstructive jaundice and underwent percutaneous transhepatic biliary drainage (PTBD) before surgery. Eleven of these patients underwent preoperative CT before PTBD, whereas 7 patients underwent $\mathrm{CT}$ after PTBD, which was performed before referral to our institution. Surgery was performed in all cases after the reduction of obstructive jaundice, and the mean interval between preoperative CT and surgery was 25.7 days (range: 7-42 days).

\section{Surgical and Histopathological Findings}

The tumors were sized and macroscopically classified based on surgical reports according to the Japanese Society of Biliary Surgery as nodular infiltrating, flat infiltrating, or papillary type.

\section{Scanning Parameters}

Preoperative CT scans using a 4-channel MDCT scanner (Lightspeed QX/I; GE Medical System, Milwaukee, WI) and nonenhanced and multiphasic contrast-enhanced scans (arterial dominant phase, portal dominant phase, and equilibrium phase) were obtained for all patients. The nonenhanced images of the upper abdomen were obtained using a detector configuration of $5 \mathrm{~mm} \times 4$ and a pitch of 3 . These images were then used to define the target volume. After the initial nonenhanced scan, $100 \mathrm{ml}(300 \mathrm{mgI} / \mathrm{ml})$ of non-ionic contrast material (Omnipaque300, Daiichi Pharmaceutical, Tokyo, Japan; Iomeron300, Eisai, Tokyo, Japan) was infused intravenously at $3 \mathrm{ml} /$ second. Arterial phase images were obtained 35 seconds after and portal phase images 70 seconds after the initiation of the injection. Both arterial and portal phase images were obtained with a small field of view (18-20 cm), a detector configuration of $1.25 \mathrm{~mm} \times 4$, a pitch of 3 , and a table speed of $3.75 \mathrm{~mm} /$ rotation at 0.8 seconds/rotation. The target volume for the arterial and portal phase images was from 1 $\mathrm{cm}$ above the umbilical portion of the left portal vein down to the ampulla of Vater. This approach provided sufficient evaluation of the secondary billiary branches of both lobes. Equilibrium phase images of the upper abdomen were initiated after a 180 -second delay with a detector configuration of $5 \mathrm{~mm} \times 4$ and a pitch of 3 .

\section{Scan Interpretation}

Three radiologists reviewed the CT images and agreed concurrently on the findings. Discrepancies were resolved by consensus. The radiologists were unaware of the surgical and pathologic findings, although they were aware of the diagnosis of cholangiocarcinoma. CT images were assessed for longitudinal tumor extension along the bile duct and for vascular invasion of the hepatic arteries and portal vein. Multiplanar reformatted (MPR) images of the arterial and portal dominant phases were reconstructed interactively during the interpretation on a workstation (Advantage Windows 4.0; GE Medical System).
Longitudinal tumor extension along the bile duct

Longitudinal tumor extension was interpreted for each segment of the bile duct tree and modified according to the classification in the Japanese Society of Biliary Surgery as follows: distal bile duct, proximal and mid bile duct, hepatic duct confluence, left hepatic duct, right hepatic duct, left-secondary biliary branches, and right-secondary biliary branches (Fig. 1).

Evidence of tumor mass, bile duct obstruction, bile-duct wall thickening, and associated contrast enhancement was evaluated. According to the imaging criteria of Lee et al. (2006), the bile duct was considered to be involved if the ductal wall was irregularly thickened, (asymmetric upstream dilatation of the intrahepatic ducts), hyperattenuated relative to the liver, obliterated the lumen, or had an intraductal soft-tissue mass. Each bile duct segment was evaluated regardless of whether it was involved by the tumor, and the overall pattern of the longitudinal extension was classified according to Bismuth-Corlette (Fig. 2).

Receiver Operating Characteristic (ROC) curve analysis was performed to test the diagnostic value of the MPR and axial images for the biliary segments. ROCIT 0.9B software (Metz CE, Chicago University) was used for the analysis. The area under each ROC curve (Az value) was used to indicate the overall performance, and a chi-square test was conducted to determine statistical differences between the ROC curves. $P<0.05$ was considered significant.

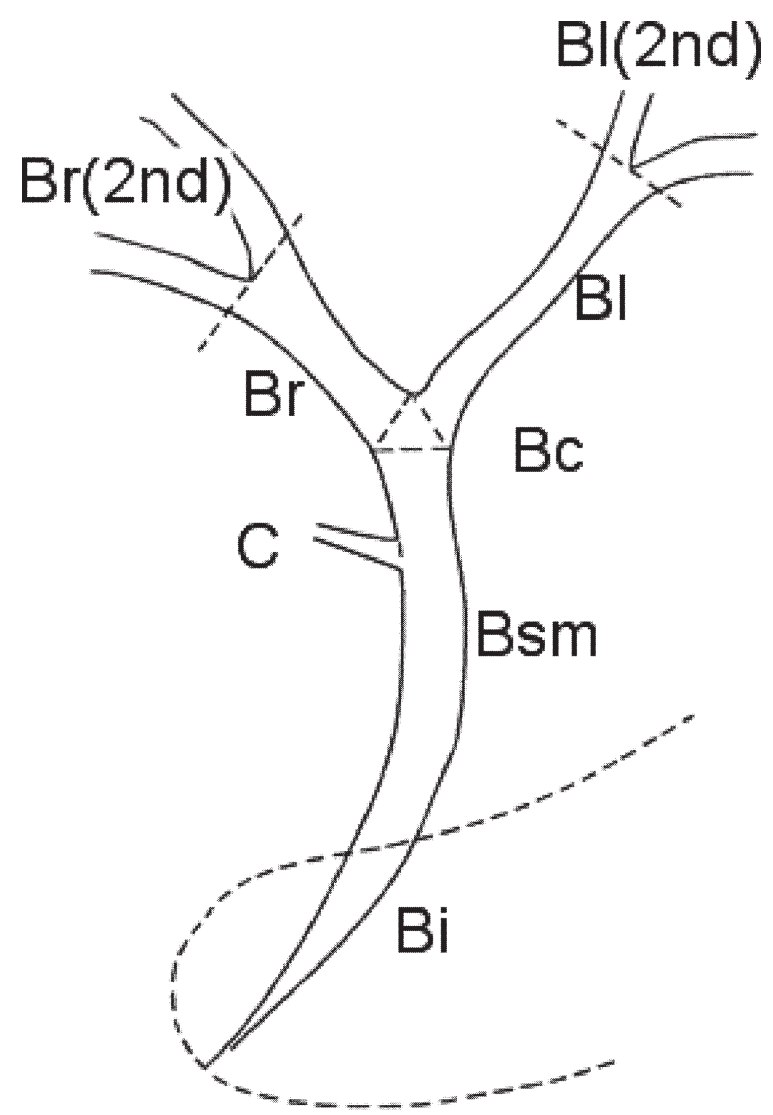

Fig. 1. Scheme for bile duct segments. Bi, intrapancreatic bile duct; Bsm, common bile duct above the pancreas and common hepatic duct; Bc, hepatic duct confluence; B1, left hepatic duct; $\mathrm{Br}$, right hepatic duct; $\mathrm{B} 12$, left secondary biliary branches; $\mathrm{Br} 2$, right secondary biliary branches; C, cystic duct. 


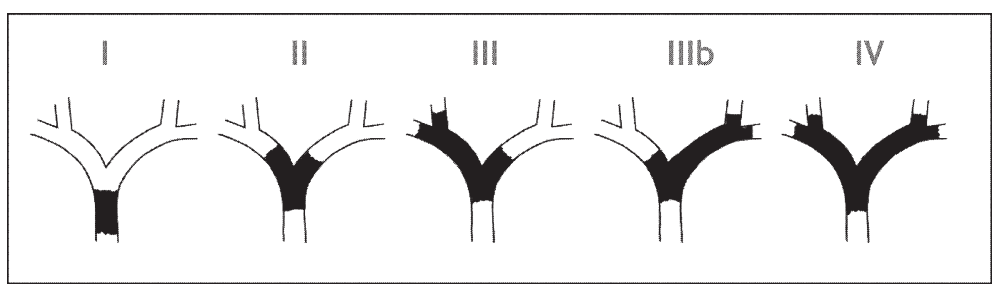

Fig. 2. Bismuth-Corlette classification of biliary obstruction. The black areas represent the bile duct segments with tumor involvement.

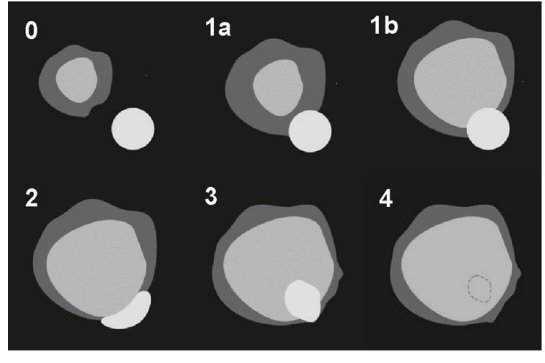

a

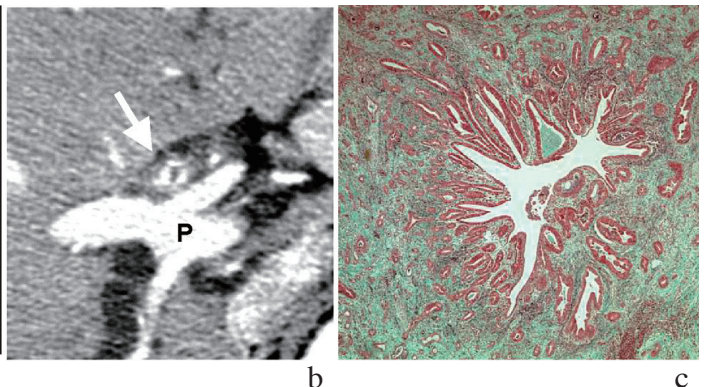

c

Fig. 3. Scheme for CT vascular invasion. (a) Schematic drawings of CT vascular-invasion grades 0-4 according to the degree of tumor $(\mathrm{T})$ contiguity with the vessel $(\mathrm{V})$. Early-central and delayed-peripheral enhancing portions of the tumor are represented as brighter central area and darker peripheral areas, respectively (b, c). Representative example of hilar cholangiocarcinoma showing the early-central enhancing pattern (arrow) and the corresponding histological specimen $(E M, 4 \times 10)$. Histological specimen shows abundant tumor cells in the luminal or central side (red stain) and corresponds to the central enhancing area of the tumor on CT, whereas the surrounding area shows more fibrosis (green stain) and relatively few tumor cells and corresponds to the less enhanced area. This case was graded as 1a against the portal vein $(\mathrm{P})$, and there was no tumor invasion on surgical examination.

\section{Vascular invasion}

The critical vessels (arteries and portal vein) of the hepatic hilar region were evaluated for surgical treatment. In cases of arterial invasion, the proper, right, and left hepatic arteries were evaluated, whereas the main portal vein and right and left primary portal venous branches were evaluated for portal vein invasion.

Vascular invasion or encasement was graded 0-4 according to the CT criteria of Baek et al. (11) [?] as follows: grade 0 (intact), when the fat plane or normal anatomical structure was visible between the tumor and the vessel; grade 1 when the fat plane between the tumor and the blood vessel was lost without displacement of the vessel; grade 2 when the vessel was displaced or narrowed by a soft tissue lesion on one side, or less than half the circumference of the vessel was in contact with the lesion; grade 3 when half or more of the circumference of the vessel was encased by a soft tissue lesion; grade 4 when a vessel was occluded by a soft tissue mass or was not visible.

Bile duct tumors often presented with early-central and delayedperipheral enhancing portions. From the relationship of the vessel with these two enhancing portions, grade 1 cases were subdivided into grades 1a and 1b: grade 1a when the vessel had no contact with the early enhancing portion of the tumor, i.e., when the two components were seen, and grade $1 \mathrm{~b}$ when the vessel had contact with the early enhancing portion of the tumor, i.e., when the two components or the vessel had contact with the tumor consisting of only a single component of enhancement (Fig. 3).

\section{Standard of Reference}

The surgical and histopathological findings were used as the gold standard. The longitudinal extension of the tumor along the bile duct and vascular invasion were determined on the basis of the histopathological findings and surgical reports.

\section{Results}

Adenocarcinomas were macroscopically classified as nodular in 12 patients and flat infiltrating in 6 patients. There was no papillary type tumor in the study group. The mean tumor size was $1.6 \mathrm{~cm}$ (range, $0.7-5.0 \mathrm{~cm}$ ). The following surgical procedures were performed: extended right hepatectomy in 8 patients, extended left hepatectomy in 5 patients, extrahepatic bile duct resection in 3 patients, and pancreatoduodenectomy in 2 patients.

\section{Comparison of longitudinal tumor extension along the bile duct by CT and pathology}

The CT, surgical, and histopathological findings for longitudinal tumor extension along the individual biliary segments along with the Bismuth-Corlette classification for bile duct extension in all 18 patients are shown in Table 1. Of the 144 biliary segments, MDTC was $96.9 \%$ sensitive and $85.1 \%$ specific for detecting, and the overall accuracy was $91.3 \%$. The accuracy for each biliary segment was distal bile duct, $94.4 \%$ (17/18); mid and proximal bile duct, $88.9 \%$ (16/18); hepatic duct confluence, 94.4\% (17/18); left hepatic duct, $88.9 \%$ (16/18); right hepatic duct, $88.9 \%$ (16/18); left secondary biliary branch, 94.4\% (17/18), and right secondary biliary branch, $83.3 \%$ (16/18). The surgical and histopathological findings showed that 7 patients had a 
TABLE 1. Comparison of CT findings with surgical and histopathological findings for longitudinal invasion along the bile duct in 18 patients.

\begin{tabular}{|c|c|c|c|c|c|c|c|c|}
\hline \multirow{2}{*}{$\begin{array}{l}\text { Patient } \\
\text { number }\end{array}$} & \multicolumn{7}{|c|}{ Biliary Segment } & \multirow{2}{*}{$\begin{array}{l}\text { Bismuth-Corlette } \\
\text { Classification }\end{array}$} \\
\hline & $\mathrm{Bi}$ & Bsm & $\mathrm{Bc}$ & $\mathrm{Br}$ & $\mathrm{Br} 2$ & $\mathrm{Bl}$ & $\mathrm{B} 12$ & \\
\hline 1 & $-\bigcirc$ & $+\bigcirc$ & $+\bigcirc$ & $+\bigcirc$ & $+\bigcirc$ & $+\bigcirc$ & +0 & IV \\
\hline 2 & $-\bigcirc$ & $+\bigcirc$ & $-\bigcirc$ & -0 & -0 & -0 & $-\bigcirc$ & $\mathrm{I} O$ \\
\hline 3 & $-\bigcirc$ & $-\bigcirc$ & $-\bigcirc$ & $+\bigcirc$ & $+\bigcirc$ & $-\bigcirc$ & $-\bigcirc$ & IIIa $\bigcirc$ \\
\hline 4 & $-\bigcirc$ & $+\bigcirc$ & $+\bigcirc$ & $+\bigcirc$ & $+\bigcirc$ & $+\bigcirc$ & $+\bigcirc$ & IV $\bigcirc$ \\
\hline 5 & $-\bigcirc$ & $-\bigcirc$ & $+\bigcirc$ & $+\bigcirc$ & $+\bigcirc$ & $+\bigcirc$ & $-\bigcirc$ & IIIa $\bigcirc$ \\
\hline 6 & $-\bigcirc$ & $+\bigcirc$ & $+\bigcirc$ & $+\bigcirc$ & $-\bigcirc$ & $+\bigcirc$ & $-\bigcirc$ & $\mathrm{II} \bigcirc$ \\
\hline 7 & $-\bigcirc$ & $+\bigcirc$ & $+\bigcirc$ & $+\bigcirc$ & $+\bigcirc$ & $+\bigcirc$ & $-\bigcirc$ & IIIa $\bigcirc$ \\
\hline 8 & $-\bigcirc$ & $+\bigcirc$ & $+\bigcirc$ & $+\bigcirc$ & $+\bigcirc$ & $+\bigcirc$ & $+\bigcirc$ & IV $\bigcirc$ \\
\hline 9 & $-\bigcirc$ & $+\bigcirc$ & $-\bigcirc$ & $-\bigcirc$ & $-\bigcirc$ & $-\bigcirc$ & $-\bigcirc$ & $\mathrm{I} \bigcirc$ \\
\hline 10 & $+\bigcirc$ & +0 & $-\bigcirc$ & $-\bigcirc$ & $-\bigcirc$ & -0 & $-\bigcirc$ & $\mathrm{I} \bigcirc$ \\
\hline 11 & -0 & $+\bigcirc$ & $+\bigcirc$ & $+\bigcirc$ & $+\bigcirc$ & $+\bigcirc$ & $-\bigcirc$ & IIIa $\bigcirc$ \\
\hline 12 & $-\bigcirc$ & $+\bigcirc$ & $+\bigcirc$ & $+\bigcirc$ & -0 & $+\bigcirc$ & $+\bigcirc$ & $\mathrm{IIIb} \bigcirc$ \\
\hline 13 & $-\bigcirc$ & $-\bigcirc$ & $+\bigcirc$ & $+\bigcirc$ & -0 & $+\bigcirc$ & $-\bigcirc$ & $\mathrm{II} \bigcirc$ \\
\hline 14 & $+\bigcirc$ & $+\bigcirc$ & -0 & $-\bigcirc$ & -0 & $-\bigcirc$ & $-\bigcirc$ & $\mathrm{I} \bigcirc$ \\
\hline 15 & $-\bigcirc$ & -0 & $+\bigcirc$ & $+\bigcirc$ & -0 & $+\bigcirc$ & $+\bigcirc$ & $\mathrm{IIIb} \bigcirc$ \\
\hline 16 & $+\bigcirc$ & $+\bigcirc$ & $-\bigcirc$ & $-\bigcirc$ & -0 & $-\bigcirc$ & $-\bigcirc$ & $\mathrm{I} \bigcirc$ \\
\hline 17 & $-\bigcirc$ & $+\bigcirc$ & $-\bigcirc$ & $-\bigcirc$ & $-\bigcirc$ & $-\bigcirc$ & $-\bigcirc$ & $\mathrm{I} \bigcirc$ \\
\hline 18 & $+\bigcirc$ & $+\bigcirc$ & +0 & -0 & -0 & -0 & $-\bigcirc$ & IO \\
\hline
\end{tabular}

type I lesion, 2 patients had a type II lesion, 4 patients had a type IIIa lesion, 2 patients had a type IIIb lesion, and 3 patients had a type IV lesion. The accuracy of CT for overall longitudinal tumor extension along the bile duct according to the Bismuth-Corlette classification was $77.8 \%$ (14/18). MPR images along the bile duct were helpful in the diagnosis of the longitudinal extension of the tumor (Figs. 4 and 5). Of the 4 cases misdiagnosed by the Bismuth-Corlette classification, 1 case was due to underestimation of the extension to the left secondary biliary branch, and 2 cases were due to overestimation of the extension to the right secondary biliary branch. Overestimation in another misdiagnosed case appeared to have resulted from the pseudo-enhancement of the bile duct wall caused by an artifact from the PTBD tube.

The ROC analysis for the evaluation of overall biliary segments indicated an $\mathrm{Az}$ value of 0.8 for axial images alone and 0.9 when evaluated using MPR images in conjunction with axial images. When the ROC curves were compared, diagnoses were significantly improved when axial and MPR images were used together $(p<0.05)$.

\section{Vascular invasion \\ Hepatic arteries}

Surgical and histopathological findings showed right hepatic artery invasion in 8 of the 18 patients. No left hepatic or proper hepatic-artery invasion was observed in the study group.
A comparison of CT findings with surgical and histopathological findings for the right hepatic artery is shown in Table 2. When grade $1 \mathrm{~b}$ and above were considered positive, the sensitivity and specificity for right hepatic arterial invasion were $100 \%$ and $90 \%$, respectively (Fig. 6), and the overall accuracy was $94.4 \%$ (17/18). In all patients, the CT findings of the left hepatic and proper hepatic arteries were correctly interpreted as grade 0 with a circumferential involvement of 0 degrees.

\section{Portal vein and branches}

Surgical and histopathological findings showed main portal vein invasion in 3 cases, right portal-vein branch invasion in 3 cases, and left portal vein branch invasion in 7 cases.

A comparison of CT findings with the surgical and histopathological findings for invasion to the three segments of the portal vein (main portal vein, right portal venous branch, and left portal venous branch) is shown in Table 3. When grade $1 \mathrm{~b}$ and above were considered positive, the overall sensitivity and specificity for portal venous invasion were $76.9 \%(10 / 13)$ and $90.2 \%$ (37/41), with overall accuracy of $87 \%(47 / 54)$.

\section{Discussion}

\section{Longitudinal tumor extension along the bile duct}

The accuracy rate of four-channel MDCT for identifying longitudinal tumor extension along the bile duct was 

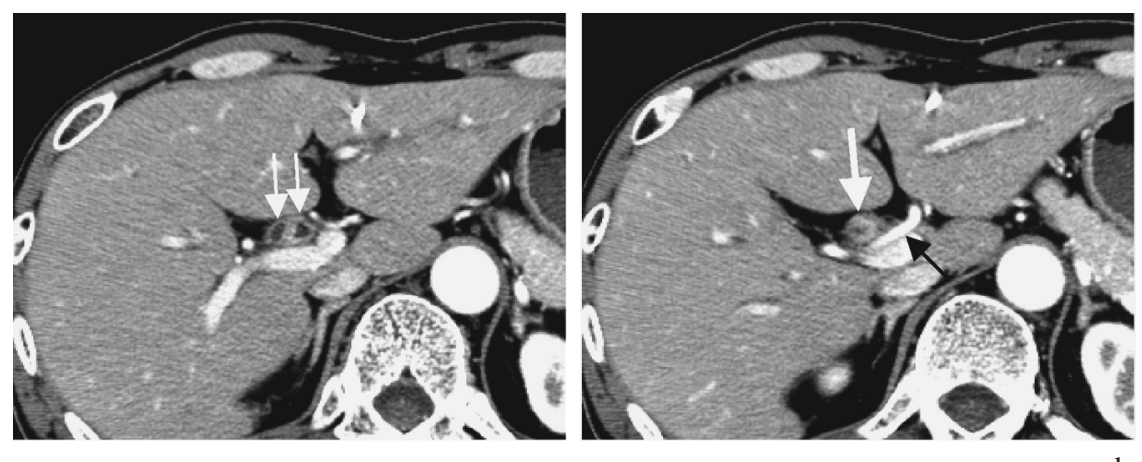

a
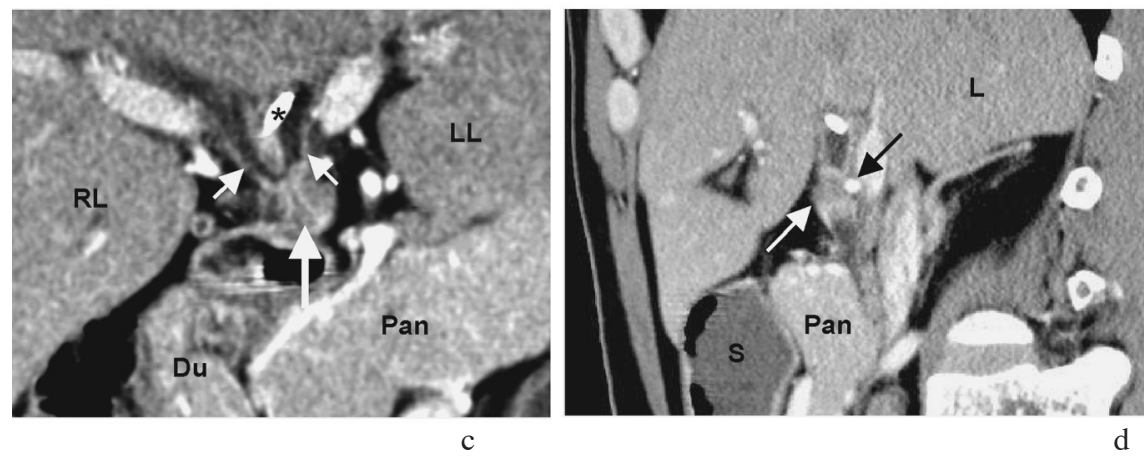

Fig. 4. A 53-year-old man with hilar cholangiocarcinoma of Bismuth-Corlette classification type 2. (a, b) Transverse arterialphase images show tumor involvement in both hepatic ducts (small white arrows), the upper bile duct (large white arrow), and the right hepatic artery (black arrow). (c) Arterial-phase oblique coronal MPR image shows the main tumor mass (large white arrow) and its longitudinal extension along both hepatic ducts (white arrows). (d) Oblique sagittal MPR image depicts contiguity of the tumor (white arrow) showing grade $1 \mathrm{~b}$ vascular involvement of the right hepatic artery (black arrow). Right hepatic artery invasion was present at the surgery. RL, right lobe of the liver; LL, left lobe of the liver; Du, duodenum; Pan, pancreas; *PTBD tube.

$77.8 \%$ (14/18), which was better than the results obtained by single-detector row helical CT (SDCT). According to a study that compared SDCT with cholangiography, SDCT had a $63 \%(17 / 27)$ overall accuracy rate for assessing biliary involvement (Han et al. 1997). In another study employing SDCT for evaluating the overall resectability of the tumor, the most common reason for inaccurate assessment was underestimation of the extent of proximal ductal tumor in 8 of 29 patients, which could be calculated as an accuracy of $70 \%$ at the maximum (Tillich et al. 1998). Our results employing MDCT were slightly better, which can be attributed to the superiority of the spatial resolution (1.25 vs. $5 \mathrm{~mm}$ in slice thickness). Another advantage of our study might have been the use of MPR images, which made it much easier to recognize the continuity of the small biliary tree (Itoh et al. 2003).

In a recent study of 55 patients that compared both SDCT ( $5 \mathrm{~mm}$ slices) and MDCT ( $2.5 \mathrm{~mm}$ slices) combined with direct cholangiography, Lee et al reported a higher diagnostic accuracy (84\%) of MDCT than SDCT for biliary involvement. However, their results may not be directly comparable with ours because direct cholangiography may have contributed substantially to the accuracy of their study (Lee et al. 2006). This procedure can be problematic because it requires injection of a contrast material through the PTBD tube that can induce cholangitis or result in an unsatisfactory delineation of the distal side of an occlusion. Neither of these may present a major problem in routine practice because a good quality direct cholangiogram can be obtained for most patients.

Recent reports of MDCT cholangiography (Lee et al. 2006; Chen et al. 2006) documented accuracies of $100 \%$ (18/18) and 91\% (10/11). This approach may be promising, although it has the same shortcomings, as mentioned for direct cholangiography. Furthermore, vascular invasion may not be completely evaluated with this method alone.

In our study, sensitivity and specificity for longitudinal tumor extension along the bile duct in 144 biliary segments were $96.9 \%$ and $85.1 \%$, respectively, with an overall accuracy of $91.3 \%$. Whether right or left lobe excision is performed during surgery depends on the presence or absence of invasion to the proximal bile ducts. Therefore, we conducted subsegment-based evaluation of bile duct invasion. Evaluation of proximal bile ducts is generally more difficult (Han et al. 1997), because these ducts are narrower and often run horizontally. However, there was no difference in the detection accuracy among proximally or distally located biliary segments, which may have been due to thinner slice thickness and the use of MPR images. In fact, longitudinal MPR sections from thin slices in conjunction with axial images helped recognize the extent of stenosis and abnormal enhancement along the bile duct. 

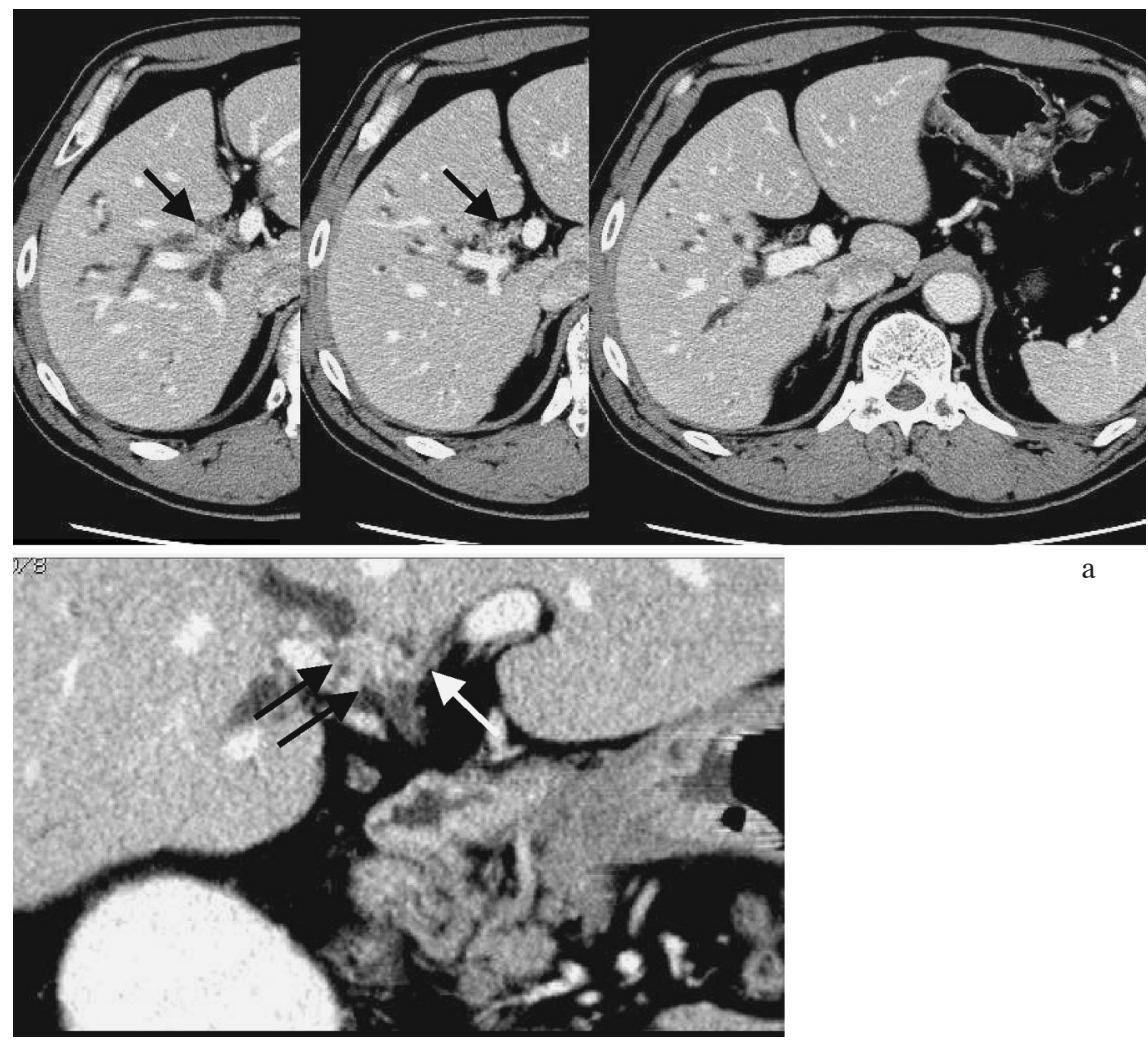

$\mathrm{b}$

Fig. 5. A 65-year-old man with hilar cholangiocarcinoma of Bismuth-Corlette classification type 3a. (a) Contiguous transverse portal-phase images show tumor mainly involving the right hepatic duct (black arrow) with dilatation of the intrahepatic ducts in the right lobe. (b) Oblique coronal MPR image depicts not only the main tumor along the right hepatic duct (black arrow) but also a slight thickening of the left hepatic duct (white arrow) that proved to be tumor extension by histopathology. Vascular invasion was not present at the surgery.

TABLE 2. Right hepatic artery invasion: comparison of CT findings with surgical and histopathological findings.

\begin{tabular}{|c|c|c|c|c|c|c|}
\hline \multirow{2}{*}{$\begin{array}{c}\text { Surgical and } \\
\text { Histopathological } \\
\text { Findings }\end{array}$} & \multicolumn{6}{|c|}{ CT grade $(n=18)$} \\
\hline & Grade 0 & Grade 1a & Grade $1 b$ & Grade 2 & Grade 3 & Grade 4 \\
\hline Invasion $(n=8)$ & & & 6 & & 2 & \\
\hline No invasion $(n=10)$ & 8 & 1 & 1 & & & \\
\hline
\end{tabular}

TABLE 3. Comparison of CT findings with surgical and histopathological findings for portal venous invasion (main portal vein, right portal venous branch, and left portal venous branch).

\begin{tabular}{lcccccc}
\hline $\begin{array}{c}\text { Surgical and } \\
\text { Histopathological } \\
\text { Findings }\end{array}$ & Grade 0 & Grade 1a & Grade 1b & Grade 2 & Grade 3 & Grade 4 \\
\cline { 2 - 6 } & & 3 & 8 & & 2 \\
Invasion $(n=13)$ & 29 & 8 & 4 & & \\
No invasion $(n=41)$ & 29 & & & \\
\hline
\end{tabular}

According to the Bismuth-Corlette classification, there were 4 cases of false results in our study. Two cases were due to overestimation in the right secondary branch and hepatic duct invasion, which may have been due to cholangitis, and one was due to overestimation of an artifact from the PTBD tube. The remaining case was due to underestimation of a left secondary branch invasion. This underesti- mation might have been due to superficial invasion along the bile duct without recognizable wall thickening and proximal bile duct dilatation due to stenosis, although this type of invasion is relatively rare in hilar cholangiocarcinoma (Sakamoto et al. 1998). Even by thin-slice CT scanning, superficial invasion may remain difficult to diagnose accurately. 


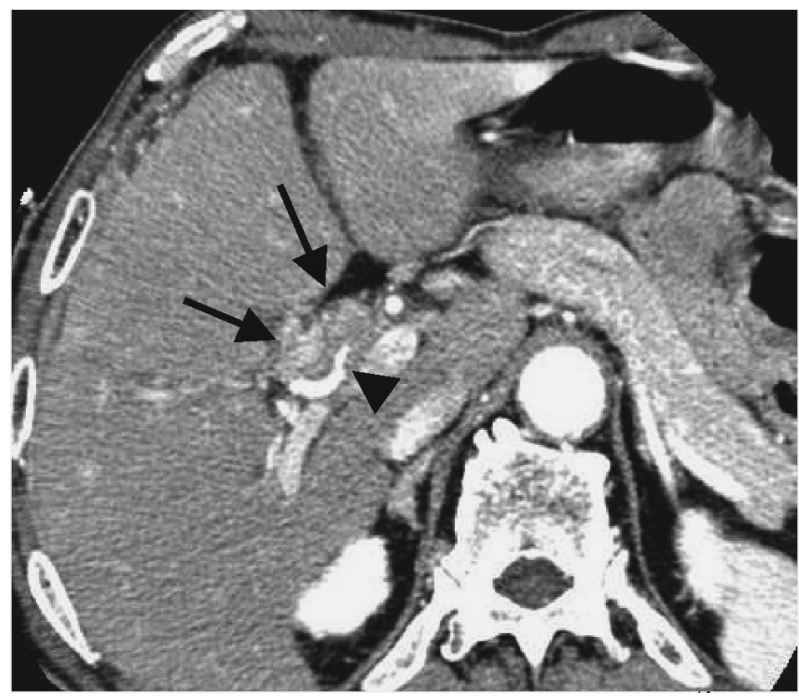

Fig. 6. A 67-year-old man with cholangiocarcinoma. Arterialphase transverse image shows involvement of the upper bile duct (arrow) with right hepatic artery invasion (arrowhead). Irregular narrowing of the artery (grade 3) is depicted.

The ROC curve analysis indicated that the diagnostic value of MDCT was significantly increased when MPR images were used in conjunction with axial images. This can be attributed to easier understanding of pathologic extension changes along the bile duct by longitudinal reformatting of the images.

\section{Vascular invasion}

Illustrative criteria for vascular invasion of hilar cholangiocarcinoma have never been established in the literature, as tumors are often small and the relevant vessels (i.e., right hepatic artery and right and left portal venous branches) do not run vertical to the axial plane. In our study, we believed that we could overcome this difficulty using MPR images in conjunction with axial images. Furthermore, we included the degree of circumferential involvement adopted for pancreatic cancer by Baek et al. (2001) as a criterion.

Only a few SDCT studies have investigated arterial invasion, but the results were poor. In an SDCT study of 11 cases with hilar cholangiocarcinoma without multiphasic dynamic contrast enhancement, 3 of 4 CT-positive patients were false positive, whereas 4 patients with actual invasion were false negatives on CT (Feydy et al. 1999). However, we found MDCT to be $100 \%$ sensitive and $80 \%$ specific for right hepatic artery invasion with an accuracy of $94.4 \%$, which is similar to a recent study (Lee et al. 2006). Along with the surgical and histopathological examinations, we correctly diagnosed the absence of left and common hepatic artery invasion in all patients by readily displaying the fat plane and normal anatomical structures. Unlike the right hepatic artery, which usually runs just behind the common hepatic bile duct, the left and common hepatic arteries are not closely attached to the bile duct at the hilar region, so they are not usually involved until a later stage of the disease.

MDCT was $76.9 \%$ sensitive, $90.2 \%$ specific, and $87.0 \%$ accurate for detecting portal vein invasion, which was also comparable with a study that used SDCT and MDCT with multiphasic scans (Lee et al. 2006).

Bile duct tumors often presented with early central and delayed peripheral enhancing portions. From the relationship of the vessel with these two enhancing portions, grade 1 cases were subdivided into grades $1 \mathrm{a}$ and $1 \mathrm{~b}$ : grade $1 \mathrm{a}$ when the vessel had no contact with the early enhancing portion of the tumor, i.e., when the two components were seen, and grade $1 \mathrm{~b}$ when the vessel had contact with the early enhancing portion of the tumor, i.e., when the two components or the vessel had contact with the tumor consisting of only a single component of enhancement (Fig. 3).

For grading vascular invasion, we subdivided grade I lesion to grades Ia and Ib. We hypothesize that grade 1a tumors have a lower probability of vascular invasion because the contact zone is a hypovascular peripheral area with relatively few tumor cells (Fig. 3), whereas grade 1 b would likely be positive for vascular invasion because the contact zone is hypervascular and more central. This hypothesis remains to be verified.

Our study had several limitations. First, we included a few patients with a PTBD tube. Because a PTBD tube relieves bile duct dilatation, it prevents the evaluation of bile duct dilatation proximal to stenosis. Other drawbacks of PTBD include artifacts adjacent to the tube and reactive bile-duct wall thickening caused by long-term insertion of the tube (Tamada et al. 1998). However, this is inevitable in a clinical setting, especially in a reference hospital, because improving obstructive jaundice is the priority for some patients at referring hospitals. Another limitation was that all of the enrolled patients had small relatively localized tumors, whereas patients with extensive invasion were excluded. In addition, the liver was only covered during non-enhanced and equilibrium phase scanning, but not arterial and portal phase scanning, which was due to technical limitations of four-channel MDCT and the risk of missing small metastatic lesions. However, this problem is resolved by newer and faster MDCT scanners, by which wider area can be scanned without sacrificing the slice thickness and image quality (i.e. scanning of the entire liver with slice thickness of $1 \mathrm{~mm})$.

In conclusion, MDCT images that are acquired with a thin-slice section in conjunction with MPR images are useful in evaluating longitudinal extension of hilar cholangiocarcinoma and detecting vascular invasion.

\section{References}

Baek, S.Y., Sheafor, D.H., Keogan, M.T., DeLong, D.M. \& Nelson, R.C. (2001) Two-dimensional multiplanar and three-dimensional volume-rendered vascular CT in pancreatic carcinoma: interobserver agreement and comparison with standard helical techniques. Am. J. Roentgenol., 176, 1467-1473. 
Chen, H.W., Pan, A.Z., Zhen, Z.J., Su, S.Y., Wang, J.H., Yu, S.C.H. \& Lau, W.Y. (2006) Preoperative evaluation of resectability of Klatskin tumor with 16-MDCT angiography and cholangiography. Am. J. Roentgenol., 186, 1580-1586.

Feydy, A., Vilgrain, V., Denys, A., Sibert, A., Belghiti, J., Vullierme, M.P. \& Menu, Y. (1999) Helical CT assessment in hilar cholangiocarcinoma: correlation with surgical and pathologic findings. Am. J. Roentgenol., 172, 73-77.

Figueras, J., Llado, L., Valls, C., Serrano, T., Ramos, E., Fabregat, J., Rafecas, A., Torras, J. \& Jaurrieta, E. (2000) Changing strategies in diagnosis and management of hilar cholangiocarcinoma. Liver Transplantation, 6, 786-794.

Han,. J.K., Choi, B.I., Kim, T.K., Kim, S.W., Han, M.C. \& Yeon, K.M. (1997) Hilar cholangiocarcinoma: thin-section spiral CT findings with cholangiographic correlation. Radiographics, 17, 1475-1485.

Hemming, A.W., Reed, A.I., Fujita, S., Foley, D.P. \& Howard, R.J. (2005) Surgical Management of Hilar Cholangiocarcinoma. Ann. Surg., 241, 693-702.

Itoh, S., Ikeda, M., Ota, T., Satake, H., Takai, K. \& Ishigaki, T. (2003) Assessment of the pancreatic and intrapancreatic bile ducts using $0.5-\mathrm{mm}$ collimation and multiplanar reformatted images in multislice CT. Eur. Radiol., 3, 277-285.

Lee, H.Y., Kim, S.H., Lee, J.M., Kim, S.W., Jang, J.Y., Han, J.K. \& Choi, B.I. (2006) Preoperative Assessment of Resectability of
Hepatic Hilar Cholangiocarcinoma: Combined CT and Cholangiography with Revised Criteria. Radiology, 239, 113-121.

Nakeeb, A., Pitt, H.A., Sohn, T.A., Coleman, J., Abrams, R.A., Piantadosi, S., Hruban, R.H., Lillemoe, K.D., Yeo, C.J. \& Cameron, J.L. (1996) Cholangiocarcinoma: a spectrum of intrahepatic, perihilar, and distal tumors. Ann. Surg., 224, 463-473

Sakamoto, E., Nimura, Y., Hayakawa, N., Kamiya, J., Kondo, S., Nagino, M., Kanai, M., Miyachi, M. \& Uesaka, K. (1998) The Pattern of infiltration at the proximal border of hilar bile duct carcinoma; A histologic analysis of 62 resected cases. Ann. Surg., 227, 405-411.

Saldinger, P.F. \& Blumgart, L.H. (2000) Resection of hilar cholangiocarcinoma - a European and United States experience. $J$. Hepatobiliary Pancreat. Surg., 7, 111-114.

Tamada, K., Tomiyama, T., Ichiyama, M., Oohashi, A., Wada, S., Nishizono, T., Tano, S., Aizawa, T., Ido, K. \& Kimura, K. (1998) Influence of biliary drainage catheter on bile duct wall thickness as measured by intraductal ultrasonography. Gastrointest. Endosc., 47, 28-32.

Tillich, M., Mischinger, H.J., Preisegger, K.H., Rabl, H. \& Szolar, D.H. (1998) Multiphasic helical CT in diagnosis and staging of hilar cholangiocarcinoma. Am. J. Roentgenol., 171, 651-658. 